PROCEEDINGS OF THE

AMERICAN MATHEMATICAL SOCIETY

Volume 139, Number 8, August 2011, Pages 2943-2955

S 0002-9939(2011)10799-8

Article electronically published on January 4, 2011

\title{
WEIGHTED SOBOLEV INEQUALITIES UNDER LOWER RICCI CURVATURE BOUNDS
}

\author{
HANS-JOACHIM HEIN
}

(Communicated by Michael Wolf)

\begin{abstract}
We obtain sharp weighted Poincaré and Sobolev inequalities over complete, noncompact Riemannian manifolds with polynomial volume growth and a quadratically decaying lower bound on Ricci. This improves and extends earlier work of Tian-Yau and Minerbe. We deduce a sharp existence result for bounded solutions of the Poisson equation on such manifolds, highlighting the well-known distinction between spaces of volume growth $\leq 2$ and $>2$ in terms of their Green's functions. We also show that if the manifold is nonparabolic and carries a smooth function which behaves like the radius function of a cone, then these solutions almost decay at the rates expected from a cone.
\end{abstract}

\section{INTRODUCTION}

Let $(M, g)$ be a complete, noncompact Riemannian manifold of dimension $n>2$. We assume throughout that $M$ satisfies a fairly mild and natural polynomial growth condition, $\operatorname{SOB}(\beta)$ (see Definition 1.1), which is supposed to capture behavior often found in Ricci-flat spaces, including the property that $\left|B\left(x_{0}, r\right)\right| \sim r^{\beta}$ for some base point $x_{0} \in M$, all $r \gg 1$, and some fixed $\beta>0$, which need not be an integer.

Our main point is to show that, under this condition, there exist global weighted Poincare and Sobolev inequalities for functions on $M$, with weights that are sharp already on simple model spaces such as flat $\mathbb{R}^{\beta} \times T^{n-\beta}$. Moreover, we demonstrate that these inequalities nicely capture some basic aspects of potential theory on $M$, in particular, the distinction between parabolic and not. More specifically, we will construct uniformly bounded solutions $u$ to $\Delta u=f$ whenever $|f| \leq C r^{-\mu}$ for some $\mu>2$ (and in addition $\int f=0$ in the parabolic case, $\beta \leq 2$ ), and we will show that $|u| \leq C_{\varepsilon} r^{2-\mu+\varepsilon}$ for every $\varepsilon>0$ if $2<\mu<\beta$ and if in addition $r$ is comparable with a smooth positive function which behaves like the radius function on a cone. This meshes well with the Green's function estimates proved by Li and Tam [13].

Being based on Moser iteration, these results also apply to other equations with a reasonable divergence structure, notably to the complex Monge-Ampère equation if $M$ is Kähler. This problem was discussed by Tian and Yau 19, 20, using various ad-hoc weighted Sobolev inequalities. For example, if $M$ is asymptotic to a minimal cone in some $\mathbb{R}^{N}$, cf. [20, they apply the Michael-Simon inequality [15] to conclude that $M$ has the same Sobolev inequality as $\mathbb{R}^{n}$. It is then natural to wonder whether the minimal surface property is responsible for this or really only the asymptotic

Received by the editors April 29, 2010 and, in revised form, July 23, 2010.

2010 Mathematics Subject Classification. Primary 53C21.

(C)2011 American Mathematical Society Reverts to public domain 28 years from publication 
conicality. Our initial motivation was to find a simple framework for dealing with such questions, and thus to strengthen the Tian-Yau existence results.

Definition 1.1. $(M, g)$ is called $\operatorname{SOB}(\beta)$ if there exist $x_{0} \in M$ and $C \geq 1$ such that $B\left(x_{0}, s\right) \backslash \bar{B}\left(x_{0}, t\right)$ is connected for all $s>t \geq C,\left|B\left(x_{0}, s\right)\right| \leq C s^{\beta}$ for all $s \geq C$, and $\left|B\left(x,\left(1-\frac{1}{C}\right) r(x)\right)\right| \geq \frac{1}{C} r(x)^{\beta}$ and $\operatorname{Ric}(x) \geq-C r(x)^{-2}$ if $r(x):=\operatorname{dist}\left(x_{0}, x\right) \geq C$.

Theorem 1.2. Suppose that $(M, g)$ satisfies $\operatorname{SOB}(\beta)$ for some $\beta \in \mathbb{R}^{+}$.

(i) For all $\varepsilon>0$ there exists a positive step function $\psi_{\varepsilon} \sim(1+r)^{-\max \{\beta, 2\}-\varepsilon}$ on $M$ such that for all $\alpha \in\left[1, \frac{n}{n-2}\right]$ and all $u \in C_{0}^{\infty}(M)$,

$$
\left(\int_{M}\left|u-u_{\varepsilon}\right|^{2 \alpha}(1+r)^{\alpha(\min \{\beta-2,0\}-\varepsilon)-\beta} d \mathrm{vol}\right)^{\frac{1}{\alpha}} \leq C_{\varepsilon} \int_{M}|\nabla u|^{2} d \mathrm{vol},
$$

where $u_{\varepsilon}$ denotes the average of $u$ with respect to the finite measure $\psi_{\varepsilon} d \mathrm{vol}$.

(ii) If $\beta>2$, then, for all $\alpha \in\left[1, \frac{n}{n-2}\right]$ and all $u \in C_{0}^{\infty}(M)$,

$$
\left(\int_{M}|u|^{2 \alpha}(1+r)^{\alpha(\beta-2)-\beta} d \mathrm{vol}\right)^{\frac{1}{\alpha}} \leq C \int_{M}|\nabla u|^{2} d \mathrm{vol} .
$$

Corollary 1.3. Let $(M, g)$ be such that there exist a compact set $K \subset M$, a compact manifold $(N, h)$, a diffeomorphism $\Phi:(1, \infty) \times N \rightarrow M \backslash K$, and $C \geq 1$ such that $\frac{1}{C} g_{\text {cone }} \leq \Phi^{*} g \leq C g_{\text {cone }}$ for the metric $g_{\text {cone }}=d t^{2} \oplus t^{2} h$ on $\mathbb{R}^{+} \times N$. Then

$$
\left(\int_{M}|u|^{2 \alpha}(1+r)^{\alpha(n-2)-n} d \mathrm{vol}\right)^{\frac{1}{\alpha}} \leq C \int_{M}|\nabla u|^{2} d \mathrm{vol}
$$

for all $u \in C_{0}^{\infty}(M)$ and $\alpha \in\left[1, \frac{n}{n-2}\right]$.

Tian-Yau [19] obtain an estimate such as (1.1) in a rather more flexible setting, but with weaker exponents that may depend on lower bounds for $|B(x, 1)|, x \rightarrow \infty$, and (1.2) overlaps with results of Minerbe [16] for Ric $\geq 0$, one of whose methods, originating from [7, we borrow. The novelty in our approach here is that we revisit foundational work on isoperimetry due to Gromov 8 to first prove a sharp Sobolev inequality with Dirichlet boundary conditions on annuli (Corollary 2.6(ii)). We can then apply a patching scheme based on [7] and [16]. Recent, independent work due to van Coevering 21] contains a proof of (1.3) via scaling and patching.

Remark 1.4. $\mathrm{SOB}(\beta)$ and (1.2) are closely related to nonparabolicity. Specifically, $\operatorname{SOB}(\beta)$ implies Li-Tam's condition (VC) from [13. Thus, from their results, $M$ is nonparabolic if and only if $\beta>2$, in which case all Green's functions are $\sim r^{2-\beta}$ at infinity. Also, from Carron [3], a complete Riemannian manifold $M$ is nonparabolic if and only if there exists $\varphi>0$ with $\int u^{2} \varphi \leq C \int|\nabla u|^{2}$ for all $u \in C_{0}^{\infty}(M)$.

Suppose in addition that $|\mathrm{Rm}| \leq C$. Thus, for all $x \in M$ there exists a smooth covering map $\Phi_{x}$ from the unit ball $B \subset \mathbb{R}^{n}$ onto a neighborhood of $x, \Phi_{x}(0)=x$, with $\frac{1}{C} g_{\mathbb{R}^{n}} \leq \Phi_{x}^{*} g \leq C g_{\mathbb{R}^{n}}$ and $\left\|\Phi_{x}^{*} g\right\|_{C^{1, \alpha}(B)} \leq C$; see Petersen [17, Theorem 4.1]. This enables us to work with the Laplacian in the global Hölder space $C^{2, \alpha}(M)$.

Theorem 1.5. Let $f \in C^{0, \alpha}(M)$ satisfy $|f| \leq C r^{-\mu}$ on $\{r>1\}$ for some $\mu>2$. If $\beta \leq 2$, then assume in addition that $\int f d \mathrm{vol}=0$. Then there exists a $u \in C^{2, \alpha}(M)$ such that $\Delta u=f$. If $\beta \leq 2$, then moreover $\int|\nabla u|^{2} d \mathrm{vol}<\infty$.

Theorem 1.6. Suppose there exists a smooth $\rho \sim 1+r$ with $|\nabla \rho|+\rho|\Delta \rho| \leq C$. If $2<\mu<\beta$, then the above solution $u$ satisfies $|u| \leq C_{\varepsilon} \rho^{2-\mu+\varepsilon}$ for all $\varepsilon>0$. 
We understand Theorems 1.5 and 1.6 as saying that the Poisson equation on $M$ largely behaves like the Poisson equation on $\mathbb{R}^{\beta}$, at least for $\beta \in \mathbb{N}$. Indeed, if $\beta>2$, then, by [13], all Green's functions $G\left(x_{0}, x\right) \sim_{x_{0}} \operatorname{dist}\left(x_{0}, x\right)^{2-\beta}$ if $\operatorname{dist}\left(x_{0}, x\right) \gg_{x_{0}} 1$, whereas in the parabolic case, $\beta \leq 2$, there exist unbounded, sign-changing Green's functions of $\log r$ and $r^{2-\beta}$ growth if $\beta=2$ and $\beta<2$, respectively. In this case, the condition $\int f=0$ is necessary to obtain a bounded solution, even if $f \in C_{0}^{\infty}(M)$.

If $M$ is Kähler of complex dimension $m$ with Kähler form $\omega$, then these theorems have close analogs for the complex Monge-Ampère equation $(\omega+i \partial \bar{\partial} u)^{m}=e^{f} \omega^{m}$. The only differences are that the lowest regularity we can deal with now is $f \in C^{2, \alpha}$ $\Rightarrow u \in C^{4, \bar{\alpha}}$ for some $\bar{\alpha} \in(0, \alpha]$ if $|\mathrm{Rm}|+\mid \nabla$ Scal $|+| \nabla^{2}$ Scal $\mid \leq C$, that the correct integrability condition to impose when $\beta \leq 2$ is $\int\left(e^{f}-1\right) \omega^{m}=0$, and that we need to assume $\rho|\partial \bar{\partial} \rho| \leq C$ in the counterpart of Theorem 1.6. Altogether, this answers, in some generality, a question raised by Tian-Yau [19, p. 581].

Example 1.7. (i) The conditions of Theorems 1.2 and 1.5 are satisfied if, outside a compact subset, $M$ is isometric to the product of a Riemannian cone of dimension $\beta \in\{2, \ldots, n\}$, or a half-line, and a closed manifold with $\mathrm{Ric} \geq 0$. If $\beta>2$, Theorem 1.6 applies as well, with $\rho$ given by the radius function on the cone factor. We refer to the Ricci-flat manifolds constructed in [11, 19] for some more involved examples of a roughly similar flavor, with $\beta \leq 2$, and $\beta \in \mathbb{Q} \backslash \mathbb{Z}$ in most cases. Their tangent cones at infinity are again Riemannian cones.

(ii) Very recently, Hattori [9] examined the geometry of complete 4-dimensional hyperkähler manifolds obtained from an infinite Gibbons-Hawking ansatz. In one of these examples, we have a 4 -manifold $M$ whose $H_{2}(M, \mathbb{Z})$ is not finitely generated. Fix $\sigma \in \mathbb{R}^{+}$. $M$ carries an $S^{1}$-action with quotient map $p: M \rightarrow \mathbb{R}^{3}$, and an explicit complete hyperkähler metric $g$ of bounded curvature, such that $p$ is a Riemannian submersion from $(M, g)$ onto $\left(\mathbb{R}^{3}, F g_{\mathbb{R}^{3}}\right)$, where $F: \mathbb{R}^{3} \rightarrow(0, \infty]$ is given by

$$
F(x)=\sum_{n \in \mathbb{Z}} \frac{1}{\left.|x-n| n\right|^{\sigma} v \mid}
$$

for some fixed unit vector $v$. The fiber $p^{-1}(x)$ has length proportional to $F(x)^{-1 / 2}$. $M$ satisfies $\operatorname{SOB}\left(4-\frac{2}{2+\sigma}\right)$ by [9, Theorem 1.1], and a candidate for $\rho$ would be

$$
\rho(x)^{2}=1+\sum_{n \in \mathbb{Z}} \frac{|x|^{2}}{|x|+|n|^{1+\sigma}} .
$$

It is easy to check that this satisfies the required bounds if $|\langle x, v\rangle| \leq \gamma|x|$ for a fixed $\gamma \in(0,1)$, but I do not know if the constants must blow up as $\gamma \rightarrow 1$. The proof of Theorem 1.6 can be localized to yield $|u| \leq C_{\varepsilon, \gamma} \rho^{2-\mu+\varepsilon}$ in this region. It would be very interesting to see whether or not this can be made independent of $\gamma$.

Notation. We write $|X|$ for the Hausdorff measure of $X \subset M$ in the right dimension, $u_{X}$ for the average and $\|u\|_{X, p}$ for the $L^{p}$ norm of $u$ over $X$ if $X$ is open, $\|u\|_{M, p}=$ $\|u\|_{p}, B=B(x, r)$ for balls, $A=A(x, r, s)$ for metric annuli, $\lambda B=B(x, \lambda r)$ if $\lambda>0$ and $\lambda A=A\left(x, \lambda^{-1} r, \lambda s\right)$ if $\lambda>1$. For $\delta \geq 0, v_{\delta}(r)=|B(r)|$ and $a_{\delta}(r)=|\partial B(r)|$ in the model space of constant curvature $-\delta^{2}$. Finally, $\alpha_{n p}=\frac{n}{n-p}$ if $1 \leq p<n$.

\section{Weighted Sobolev inequalities}

The key to the proof is a volume comparison method introduced by Gromov [8]. Buser [2, Maheux and Saloff-Coste [14, and Cheeger-Colding [4] have applied this 
method to derive various $L^{p}$ Poincaré and Sobolev inequalities of Neumann type, for the most part on geodesic balls, and there is a Dirichlet-type $L^{1}$ Sobolev inequality for geodesic balls, due to Anderson [1], based on the same principle. In Sections 2.1 and 2.2, we show that the same idea also yields Dirichlet-type inequalities on more general domains, especially on annuli (Corollary 2.6). This quickly yields a global Sobolev inequality under lower Ricci bounds (Corollary 2.8), which implies Gallot's inequality [6] on compact manifolds. In Section 2.3, we then combine Corollary 2.6 with patching methods from Grigor'yan and Saloff-Coste 7] and Minerbe [16, and with the Cheeger-Colding segment inequality [4, to prove Theorem 1.2 .

2.1. Isoperimetric estimates à la Gromov and Anderson. Let $M$ denote an $n$-dimensional Riemannian manifold without boundary, which may be incomplete. The goal of this section is to prove a general estimate (Lemma 2.2) for the volume of a domain in $M$ in terms of the area of its boundary, based on an idea of Gromov [8. §6.C, Appendix C] and Anderson [1, Section 4].

Definition 2.1. Let $X$ be a metric-measure space, $Y \subset X$. Let $\mathcal{B}$ be a covering of $Y$ by metric balls. We say that $\mathcal{B}$ is $\left(\varepsilon, r_{0}\right)$-good, $0<\varepsilon \leq \frac{1}{2}, r_{0}>0$, if center $(B) \in Y$, $\min \{|B \backslash Y|,|B \cap Y|\} \geq \varepsilon|B|$, and $\operatorname{radius}(B) \leq r_{0}$ for all $B \in \mathcal{B}$.

Lemma 2.2. Let $\Omega \subset M$ be open with a smooth boundary. Let $\mathcal{B}$ be an $\left(\varepsilon, r_{0}\right)$-good covering of $\Omega$. Let $N$ be the open $5 r_{0}$-neighborhood of $\Omega$, and assume $\bar{N}$ is compact. If Ric $\geq-(n-1) \delta^{2}$ on $N$ with $0 \leq r_{0} \delta \leq \Lambda$, and if $\alpha \geq 1$, then

$$
\frac{|\Omega|^{\frac{1}{\alpha}}}{|\partial \Omega|} \leq C(n, \varepsilon, \Lambda) \sup _{B \in \mathcal{B}}|B|^{\frac{1}{\alpha}-1} \operatorname{radius}(B) .
$$

Proof. Choose finitely many balls $B_{i}=B\left(x_{i}, r_{i}\right) \in \mathcal{B}, i=1, \ldots, k$, such that the $2 B_{i}$ are pairwise disjoint but still $\Omega \subset \bigcup 5 B_{i}$. This can be achieved through a standard Vitali type procedure: Since $\bar{\Omega}$ is compact and $B \backslash \Omega \neq \emptyset$ for all $B \in \mathcal{B}$, there exists a finite subcollection $\mathcal{B}^{\prime} \subset \mathcal{B}$ that still covers $\Omega$. We choose a $B_{1} \in \mathcal{B}^{\prime}$ of maximal radius, and we take $B_{i+1} \in \mathcal{B}^{\prime}$ to be of maximal radius among all those $B \in \mathcal{B}^{\prime}$ for which $2 B$ is disjoint from $2 B_{1}, \ldots, 2 B_{i}$. Then, indeed, $\bigcup 5 B_{i} \supset \bigcup \mathcal{B}^{\prime} \supset \Omega$.

Key estimate: We have $\left|B_{i}\right| \leq C(n, \varepsilon, \Lambda) r_{i}\left|\partial \Omega \cap 2 B_{i}\right|$ for all $i=1, \ldots, k$.

If this is true, then (2.1) follows immediately by noting that

$$
|\Omega|^{\frac{1}{\alpha}} \leq C(n, \Lambda) \sum\left|B_{i}\right|^{\frac{1}{\alpha}} \leq C(n, \varepsilon, \Lambda) \sum\left|B_{i}\right|^{\frac{1}{\alpha}-1} r_{i}\left|\partial \Omega \cap 2 B_{i}\right| .
$$

Proof of the key estimate. The basic idea is as follows. Fix $z \in B_{i} \cap \Omega$ and project $B_{i} \backslash \Omega$ onto $\partial \Omega$ along minimal geodesics emanating from $z$. Define $\Sigma^{\text {first }} \subset \partial \Omega \cap 2 B_{i}$ to consist of the first points of entry into $B_{i} \backslash \Omega$ of the "light rays" involved in this projection. By integrating the infinitesimal version of the Bishop-Gromov volume comparison inequality along the maximal subsegment with endpoints in $\bar{B}_{i} \backslash \Omega$ of each such light ray, and then integrating across $\Sigma^{\text {first }}$, one eventually finds that

$$
\varepsilon\left|B_{i}\right| \leq\left|B_{i} \backslash \Omega\right| \leq \int_{\Sigma_{\text {first }}} \frac{v_{\delta}\left(2 r_{i}\right)-v_{\delta}(\operatorname{dist}(z, y))}{a_{\delta}(\operatorname{dist}(z, y))} \operatorname{darea}(y) .
$$

This proves the key estimate if $r_{i} \leq C(n, \varepsilon, \Lambda) \operatorname{dist}\left(z, \Sigma^{\mathrm{first}}\right)$. But if this inequality fails for all $z \in B_{i} \cap \Omega$, then intuitively one should be able to find points $z^{\prime} \in B_{i} \backslash \Omega$ such that the argument does go through with $z, B_{i} \backslash \Omega$ replaced by $z^{\prime}, B_{i} \cap \Omega$.

We now work out the details. Specifically, we show that $\min \left\{\left|B_{i} \backslash \Omega\right|,\left|B_{i} \cap \Omega\right|\right\} \leq$ $2\left(v_{\delta}\left(4 r_{i}\right) / a_{\delta}\left(2 r_{i}\right)\right)\left|\partial \Omega \cap 2 B_{i}\right|$, and this then suffices by $\left(\varepsilon, r_{0}\right)$-goodness. 
Define $X_{1}, X_{2} \subset\left(B_{i} \cap \Omega\right) \times\left(B_{i} \backslash \Omega\right)$ as follows: $X_{1}:=\left\{\left(z, z^{\prime}\right)\right.$ : there is a unique minimal geodesic $\gamma$ from $z$ to $z^{\prime}$, and this has the following properties: it intersects $\partial \Omega$ only transversely, and if $y$ denotes the first point along $\gamma$, counted from $z$, where $\gamma$ intersects $\partial \Omega$, then $\left.\operatorname{dist}(z, y) \geq \operatorname{dist}\left(y, z^{\prime}\right)\right\}$, and almost verbatim for $X_{2}$, with the only difference that the inequality is now reversed. Then $X_{1} \cup X_{2}$ has full measure in $\left(B_{i} \cap \Omega\right) \times\left(B_{i} \backslash \Omega\right)$, and so one of $X_{1}, X_{2}$ must have at least half measure.

- $X_{1}$ has at least half measure. By Fubini, there must be a $z \in B_{i} \cap \Omega$ such that $Z^{\prime}:=\left\{z^{\prime} \in B_{i} \backslash \Omega:\left(z, z^{\prime}\right) \in X_{1}\right\}$ has at least half measure in $B_{i} \backslash \Omega$. We now bound $\left|Z^{\prime}\right|$ above by projecting onto $\partial \Omega$ along minimal geodesics from $z$, and integrating the infinitesimal Bishop-Gromov inequality along these geodesics.

Let $\Sigma^{\text {first }}$ be the set of all $y \in \partial \Omega \cap 2 B_{i}$ which occur as the first intersection with $\partial \Omega$ of the geodesic $\gamma$ from $z$ to some $z^{\prime} \in Z^{\prime}$ as in the definition of $X_{1}$. Thus, for all $y \in \Sigma^{\text {first }}$ there exists a unique minimal geodesic $\gamma_{y}$ from $z$ to $y$, and we can write $\gamma_{y}(t)=\exp _{z}\left(v_{y} t\right)$, where $v_{y} \in T_{z} M$ is a uniquely determined unit vector.

Define $d_{1}, d_{2}: \Sigma^{\text {first }} \rightarrow \mathbb{R}^{+}$by $d_{1}(y):=\operatorname{dist}(z, y)$ and $d_{2}(y):=\min \{\sup \{t>0$ : $\left.\gamma_{y}\left(d_{1}(y)+t\right) \in Z^{\prime}\right\}, \sup \left\{t>0: \gamma_{y}\right.$ is minimal on $\left.\left.\left[0, d_{1}(y)+t\right]\right\}\right\}$.

For every $z^{\prime} \in Z^{\prime}$ then, there exists a unique $y \in \Sigma^{\text {first }}$ such that $z^{\prime}=\gamma_{y}(t)$ for some $d_{1}(y)<t \leq d_{1}(y)+d_{2}(y)$. Thus, if we define an imbedding $\Phi: U \hookrightarrow M$ with $U:=\left\{(y, t) \in \Sigma^{\text {first }} \times \mathbb{R}^{+}: d_{1}(y)<t<d_{1}(y)+d_{2}(y)\right\}$ and $\Phi(y, t):=\gamma_{y}(t)$, then $\operatorname{clos}(\Phi(U))$ contains $Z^{\prime}$. On the other hand, a fairly standard calculation yields

$$
\left.\Phi^{*}\left(d \operatorname{vol}_{M}\right)\right|_{(y, t)}=\frac{J\left(t v_{y}\right)}{J\left(d_{1}(y) v_{y}\right)} \cos \alpha_{y} d \operatorname{area}_{\partial \Omega} \wedge d t,
$$

where $J(w):=\left.|w|^{n-1} \operatorname{det} d \exp _{z}\right|_{w}$ for all $w \in T_{z} M$, and where $\alpha_{y}$ denotes the angle between $\dot{\gamma}_{y}\left(d_{1}(y)\right)$ and the exterior unit normal to $\partial \Omega$ at $y$.

We integrate over $U$ and apply relative volume comparison:

$$
\begin{aligned}
|\Phi(U)| & \leq \int_{\Sigma^{\mathrm{first}}} \int_{d_{1}(y)}^{d_{1}(y)+d_{2}(y)} \frac{J\left(t v_{y}\right)}{J\left(d_{1}(y) v_{y}\right)} d t \text { darea }(y) \\
& \leq \int_{\Sigma^{\mathrm{first}}} \int_{d_{1}(y)}^{d_{1}(y)+d_{2}(y)} \frac{a_{\delta}(t)}{a_{\delta}\left(d_{1}(y)\right)} d t \text { darea }(y) \\
& =\int_{\Sigma^{\mathrm{first}}} \frac{v_{\delta}\left(d_{1}(y)+d_{2}(y)\right)-v_{\delta}\left(d_{1}(y)\right)}{a_{\delta}\left(d_{1}(y)\right)} d \operatorname{darea}(y) .
\end{aligned}
$$

We now estimate the integrand as follows:

$$
\frac{v_{\delta}\left(d_{1}+d_{2}\right)-v_{\delta}\left(d_{1}\right)}{a_{\delta}\left(d_{1}\right)} \leq \frac{v_{\delta}\left(d_{1}+d_{2}\right)}{a_{\delta}\left(d_{1}\right)} \leq \frac{v_{\delta}\left(2 d_{1}\right)}{a_{\delta}\left(d_{1}\right)} \leq \frac{v_{\delta}\left(4 r_{i}\right)}{a_{\delta}\left(2 r_{i}\right)},
$$

because $d_{2} \leq d_{1} \leq 2 r_{i}$ and because $s \mapsto v_{\delta}(2 s) / a_{\delta}(s)$ is nondecreasing. Altogether then, $\left|B_{i} \backslash \Omega\right| \leq 2\left|Z^{\prime}\right| \leq 2|\Phi(U)| \leq 2\left(v_{\delta}\left(4 r_{i}\right) / a_{\delta}\left(2 r_{i}\right)\right)\left|\Sigma^{\text {first }}\right|$, as needed.

- $X_{2}$ has at least half measure. By Fubini again, there now exists a $z^{\prime} \in B_{i} \backslash \Omega$ such that $Z:=\left\{z \in B_{i} \cap \Omega:\left(z, z^{\prime}\right) \in X_{2}\right\}$ has at least half measure in $B_{i} \cap \Omega$. Let $\Sigma^{\text {last }}$ denote the set of all $y \in \partial \Omega \cap 2 B_{i}$ which occur as the last intersection of $\gamma^{-1}$ with $\partial \Omega$, where $\gamma$ is the geodesic from some $z \in Z$ to $z^{\prime}$ as in the definition of $X_{2}$. For each $y \in \Sigma^{\text {last }}$, there then exists a unique minimal geodesic $\gamma_{y}(t)=\exp _{z^{\prime}}\left(t v_{y}\right)$ from $z^{\prime}$ to $y$, and the rest of the argument will be the same as above up to replacing $z, Z^{\prime}, \Sigma^{\text {first }}$ by $z^{\prime}, Z, \Sigma^{\text {last }}$ and switching the interior and exterior normals of $\Omega$.

Remark 2.3. A similar sort of reasoning yields the following result: If $B=B(x, r) \subset$ $M$ is such that $3 r<\operatorname{diam}(M), 4 \bar{B}$ is compact, and Ric $\geq-\Lambda r^{-2}$ on $4 B$ with $\Lambda \geq 0$, 
then $\frac{1}{C} r|\partial B| \leq|B| \leq C r|\partial B|$ with a uniform $C=C(n, \Lambda)$ which in particular does not depend on the collapsedness of $B$. Here, the upper bound follows as before, by projecting $B$ onto $\partial B$ along minimal geodesics issuing from a point on $\partial(3 B)$. For the lower bound, we sweep out a subset $B^{*} \subset B$ by joining $x$ to all smooth points of $\partial B$, express $\left|B^{*}\right|$ in polar coordinates, and then use Bishop-Gromov in the form $J(t v) / J(r v) \geq a_{\delta}(t) / a_{\delta}(r), v \in T_{x} M,|v|=1, t \leq r$, to estimate from below.

2.2. Dirichlet-type Sobolev inequalities on balls and annuli. Under lower Ricci bounds, subsets of geodesic balls or annuli admit $\left(\varepsilon, r_{0}\right)$-good ball coverings for controlled values of $\varepsilon, r_{0}$ (Lemma 2.4). By Lemma 2.2, this implies a Dirichlet-isoperimetric, hence a Dirichlet-Sobolev inequality (Corollary 2.6). As a corollary, we obtain a global Gallot- or Varopoulos-type inequality (Corollary 2.8).

Note that $M$ is still not required to be complete. In the following lemma, we fix a point $x_{0} \in M$, and we put $B(r):=B\left(x_{0}, r\right), A\left(r_{1}, r_{2}\right):=A\left(x_{0}, r_{1}, r_{2}\right)$.

Lemma 2.4. (i) For all $\Lambda \geq 0$ there exists $\varepsilon=\varepsilon(n, \Lambda)>0$ such that if $B:=B(s)$, $4 s<\operatorname{diam}(M), 9 \bar{B}$ is compact, and Ric $\geq-\Lambda s^{-2}$ on $9 B$, then for all open $\Omega \subset B$ and $x \in \Omega$ there exists $0<r_{x, \Omega} \leq 4 s$ such that $\left|B\left(x, r_{x, \Omega}\right) \backslash \Omega\right|=\varepsilon\left|B\left(x, r_{x, \Omega}\right)\right|$.

(ii) For all $\Lambda, N \geq 0$ there exists $\varepsilon=\varepsilon(n, \Lambda, N)>0$ such that if $A:=A(r, r+s)$, $r>6 t, s \leq N t, \bar{B}(r+2 s+2 t)$ is compact, and Ric $\geq-\Lambda t^{-2}$ on $A(r-6 t, r+2 s+2 t)$, then for all open $\Omega \subset A$ and $x \in \Omega$ there exists $0<r_{x, \Omega} \leq r_{x}^{*}:=\operatorname{dist}\left(x_{0}, x\right)-r+2 t$ such that $\left|B\left(x, r_{x, \Omega}\right) \backslash \Omega\right|=\varepsilon\left|B\left(x, r_{x, \Omega}\right)\right|$.

Proof. (i) Fix a minimal geodesic $\gamma$ from $x$ to some point $x^{\prime \prime} \in \partial B(x, 4 s)$ and put $x^{\prime}:=\gamma(3 s)$. Then, by volume comparison, $|B(x, 4 s) \backslash B| \geq\left|B\left(x^{\prime}, s\right)\right| \geq \varepsilon|B(x, 4 s)|$ for some definite $\varepsilon=\varepsilon(n, \Lambda)>0$. The claim then follows by continuity.

(ii) Fix a minimal geodesic $\gamma$ from $x_{0}$ to $x$. Let $x_{i}:=\gamma(r+(2 i-3) t), B_{i}:=B\left(x_{i}, t\right)$ for $i=1, \ldots, k$, where $k$ is maximal with $r+(2 k-3) t \leq \operatorname{dist}\left(x_{0}, x\right)$; notice that $k \leq \frac{1}{2}(N+3)$. Then $B\left(x, r_{x}^{*}\right) \backslash A \supset B_{1}$ and $3 B_{i} \supset B_{i+1}$ for $i=1, \ldots, k-1$. A volume comparison shows that $\left|B_{i}\right| \geq \varepsilon\left|3 B_{i}\right|$; thus $\left|B\left(x, r_{x}^{*}\right) \backslash A\right| \geq \varepsilon\left|B_{k}\right|$ by induction, where we understand $\varepsilon$ as a small generic constant. By volume comparison again, $\left|B_{k}\right| \geq$ $\varepsilon\left|B\left(x_{k}, 2 t+r_{x}^{*}\right)\right|$, and $B\left(x_{k}, 2 t+r_{x}^{*}\right) \supset B\left(x, r_{x}^{*}\right)$ by maximality of $k$. Altogether, $\left|B\left(x, r_{x}^{*}\right) \backslash A\right| \geq \varepsilon\left|B\left(x, r_{x}^{*}\right)\right|$, so we conclude as in (i).

Thus, for all $\Omega \subset B, A$ in (i), (ii), the covering $\left\{B\left(x, r_{x, \Omega}\right): x \in \Omega\right\}$ is $\left(\varepsilon, r_{0}\right)$-good with $\varepsilon=\varepsilon(n, \Lambda), r_{0}=4 s$, and $\varepsilon=\varepsilon(n, \Lambda, N), r_{0}=s+2 t$, respectively, so then Lemma 2.2 provides a uniform isoperimetric estimate for all such $\Omega$. It is a classical fact that this implies Dirichlet-Sobolev inequalities on $B, A$; cf. Li [12, Theorem 9.1] and Saloff-Coste [18, Section 3.1.2] for expositions. We recall the relevant result for convenience, and then state the ensuing Sobolev bounds in our setting.

Lemma 2.5. For all $\Omega_{0} \subset M$ open and precompact, $\alpha \geq 1$,

$$
\sup \left\{\frac{\|u\|_{\alpha}}{\|\nabla u\|_{1}}: u \in C_{0}^{\infty}\left(\Omega_{0}\right), u \neq 0\right\}=\sup \left\{\frac{|\Omega|^{\frac{1}{\alpha}}}{|\partial \Omega|}: \Omega \subset \Omega_{0} \text { open, } \partial \Omega \text { smooth }\right\} .
$$

Also, recall that by Hölder's inequality, for all $p \geq 1$ and $\alpha \geq 1$,

$$
\operatorname{DS}\left(\Omega_{0}, p, \alpha\right):=\sup \frac{\|u\|_{\alpha p}}{\|\nabla u\|_{p}} \leq \frac{\alpha p}{\alpha^{\prime}} \sup \frac{\|u\|_{\alpha^{\prime}}}{\|\nabla u\|_{1}} \text { if } 1-\frac{1}{\alpha^{\prime}}=\frac{1}{p}\left(1-\frac{1}{\alpha}\right),
$$

where the suprema are over all $u \in C_{0}^{\infty}\left(\Omega_{0}\right)$ with $u \neq 0$. 
Corollary 2.6. (i) If $20 s<\operatorname{diam}(M)$ and $\bar{B}(20 s)$ is compact, and if $\operatorname{Ric} \geq-\Lambda s^{-2}$ on $B(20 s)$ with $\Lambda \geq 0$, then, for all $p \in[1, n)$ and $\alpha \in\left[1, \alpha_{n p}\right]$,

$$
\operatorname{DS}(B(s), p, \alpha) \leq C(n, p, \Lambda) s|B(s)|^{\frac{1}{p}\left(\frac{1}{\alpha}-1\right)} .
$$

(ii) If $20 s<\min \{r, \operatorname{diam}(M)\}$ and $\bar{B}(r+20 s)$ is compact, and if $\operatorname{Ric} \geq-\Lambda s^{-2}$ on $A(r-20 s, r+20 s)$ with $\Lambda \geq 0$, then, for all $p \in[1, n)$ and $\alpha \in\left[1, \alpha_{n p}\right]$,

$$
\operatorname{DS}(A(r, r+s), p, \alpha) \leq C(n, p, \Lambda) s \sup _{x \in A(r, r+s)}|B(x, s)|^{\frac{1}{p}\left(\frac{1}{\alpha}-1\right)} .
$$

Proof. (i) Combining Lemmas 2.2, 2.4, with Lemma 2.5, (2.2),

$$
\operatorname{DS}(B(s), p, \alpha) \leq C(n, p, \alpha, \Lambda) \sup \left\{r|B(x, r)|^{\frac{1}{p}\left(\frac{1}{\alpha}-1\right)}: x \in B(s), r \in(0,4 s]\right\}
$$

for all $p \geq 1, \alpha \geq 1$. The right-hand side is finite iff either $p \in[1, n)$ and $\alpha \in\left[1, \alpha_{n p}\right]$, or $p \geq n$. The second case is covered by the first, and in the first case, (2.3) follows by volume comparison. The proof of (ii) is similar.

Remark 2.7. Corollary 2.6(i) reproduces Anderson [1, Theorem 4.1], which has its roots in Gromov [8]. We have streamlined Anderson's proof and tweaked it so that it applies to more general domains. Note that (2.3) simply means that if $B=B(s)$, then, for all $\alpha \in\left[1, \alpha_{n p}\right]$ and all $u \in C_{0}^{\infty}(B)$,

$$
\left(f_{B}|u|^{\alpha p}\right)^{\frac{1}{\alpha p}} \leq C(n, p, \Lambda) s\left(f_{B}|\nabla u|^{p}\right)^{\frac{1}{p}} \text {. }
$$

Croke's sharp isoperimetric inequality from [5] would imply (2.5) with an additional collapsedness factor of $s^{n}|B|^{-1}$ on the right; the improvement afforded by (2.5) was crucial for the main $\varepsilon$-regularity theorem proved in 11. By Maheux and Saloff-Coste [14, Théorème 1.1], (2.5) holds as well for all $u \in C^{\infty}(B)$ with mean value zero.

We conclude with a global Sobolev inequality similar to the ones given in Hebey [10. Theorem 3.14, Theorem 3.22] which follows immediately from Corollary 2.6. In the compact case, this implies a familiar result of Gallot [6, Théorème 6.16].

Corollary 2.8. Let $M^{n}$ be a complete Riemannian manifold. If $r, \varepsilon>0$ and $\Lambda \geq 0$ are such that $100 r<\operatorname{diam}(M)$, Ric $\geq-\Lambda r^{-2}$, and $|B(x, r)| \geq \varepsilon$ for all $x \in M$, and if $p \in[1, n)$ and $\alpha \in\left[1, \alpha_{n p}\right]$, then

$$
\|u\|_{\alpha p} \leq C(n, p, \Lambda) \varepsilon^{\frac{1}{p}\left(\frac{1}{\alpha}-1\right)}\left(r\|\nabla u\|_{p}+\|u\|_{p}\right),
$$

for all $u \in C_{0}^{\infty}(M)$ if $M$ is open, and for all $u \in C^{\infty}(M)$ if $M$ is closed.

Proof. Fix $x_{0} \in M$ and define $r_{m}:=\left(1+\frac{m}{100}\right) r, A_{m}:=A\left(x_{0}, r_{m}, r_{m+1}\right)$ for $m \in \mathbb{N}_{0}$. Take $\chi_{0} \in C_{0}^{\infty}\left(B\left(x_{0}, r_{2}\right)\right)$ with $0 \leq \chi_{0} \leq 1, \chi_{0} \equiv 1$ on $B\left(x_{0}, r_{1}\right),\left|\nabla \chi_{0}\right| \leq 200 r^{-1}$. For $m \geq 1$ take $\chi_{m} \in C_{0}^{\infty}\left(A_{m-1} \cup \bar{A}_{m} \cup A_{m+1}\right)$ with $0 \leq \chi_{m} \leq 1, \chi_{m} \equiv 1$ on $A_{m}$, $\left|\nabla \chi_{m}\right| \leq 200 r^{-1}$. By Corollary 2.6 for all $m \in \mathbb{N}_{0}$,

$$
\left\|u \chi_{m}\right\|_{\alpha p} \leq C(n, p, \Lambda) r \varepsilon^{\frac{1}{p}\left(\frac{1}{\alpha}-1\right)}\left\|\nabla\left(u \chi_{m}\right)\right\|_{p} .
$$

Take $p$-th powers, sum over $m$, and take $p$-th roots. 
2.3. Proof of the weighted Sobolev inequalities. This section concludes the proof of Theorem 1.2 by combining Corollary 2.6 and some basic analysis on graphs, similar to what was developed in [7, 16] in much greater generality.

The key step in passing from the Dirichlet-Sobolev estimates in Corollary 2.6 to the global estimates in Theorem 1.2 is a certain Neumann-type Poincaré inequality. We need both a continuous and a discrete version. The continuous one follows from a special case of the Cheeger-Colding segment inequality [4, Theorem 2.11]:

Lemma 2.9 (Cheeger-Colding). Let $B=B(x, r) \subset\left(M^{n}, g\right)$. If $2 B$ is precompact and Ric $\geq-\Lambda r^{-2}$ on $2 B$ with $\Lambda \geq 0$, then, for all $u \in C^{\infty}(B)$,

$$
\int_{B}\left|u-u_{B}\right|^{2} \leq C(n, \Lambda) r^{2} \int_{2 B}|\nabla u|^{2}
$$

Passing from $B$ to $2 B$ is inevitable in their proof because a segment between two points in $B$ will usually only be contained in $2 B$. Buser [2, Lemma 5.1] gives an $L^{p}$ Neumann-type Poincaré inequality for every $p \geq 1$ which does not require doubling the radius, but (2.7) yields all we need here and is surprisingly simple to show.

Also, (2.7) has a useful discrete counterpart with a closely related proof. If $V$ is a set and $E$ is a set of 2-element subsets of $V$, both countable, we call $G=(V, E)$ a graph. We say $G$ is connected if any two vertices $x, y \in V, x \neq y$, can be joined by a path, i.e. a set $\gamma \subset V$ which can be listed as $\gamma=\left\{\gamma_{0}, \ldots, \gamma_{m}\right\}$ such that $\left\{\gamma_{0}, \gamma_{m}\right\}=$ $\{x, y\}$ and $\left\{\gamma_{i-1}, \gamma_{i}\right\} \in E$ for $i=1, \ldots, m$. If $u: V \rightarrow \mathbb{C}$, then we write $u_{x}:=u(x)$, and we define $|\nabla u|^{2}: V \rightarrow \mathbb{R}$ by setting $|\nabla u|_{x}^{2}:=\sum_{y \in V:\{x, y\} \in E}\left|u_{x}-u_{y}\right|^{2}$.

Lemma 2.10. Let $G$ be connected and let $w: V \rightarrow \mathbb{R}^{+}$satisfy $\sum w_{x}=1$. For all $x, y \in V, x \neq y$, fix a path $\gamma=\gamma^{\{x, y\}}$ as above, and write $m=m^{\{x, y\}}$. Introduce $\bar{w}: V \rightarrow \mathbb{R}^{+}$as $\bar{w}_{z}:=\sum_{\{x, y\}: z \in \gamma^{\{x, y\}}} m^{\{x, y\}} w_{x} w_{y}$. Then, for all $u: V \rightarrow \mathbb{C}$,

$$
\sum_{x \in V} w_{x} u_{x}=0 \Longrightarrow \sum_{x \in V} w_{x}\left|u_{x}\right|^{2} \leq \sum_{x \in V} \bar{w}_{x}|\nabla u|_{x}^{2} .
$$

Indeed, multiply the left-hand side by $\sum w_{x}=1$ and use $\sum w_{x} u_{x}=0$ to obtain

$$
\sum_{x} w_{x}\left|u_{x}\right|^{2}=\sum_{\{x, y\}} w_{x} w_{y}\left|u_{x}-u_{y}\right|^{2} \leq \sum_{\{x, y\}} m^{\{x, y\}} w_{x} w_{y} \sum_{i=1}^{m^{\{x, y\}}}\left|u_{\gamma_{i}^{\{x, y\}}}-u_{\gamma_{i-1}^{\{x, y\}}}\right|^{2} .
$$

Proof of Theorem 1.2. During this proof, both $C_{0}$ and $C$ will denote large constants that are only allowed to depend on the geometry of $M$, but not on the function $u$. However, $C_{0}$ is fixed once and for all, whereas $C$ may change from line to line.

Fix $\alpha \in\left[1, \alpha_{n 2}\right]$ and let $\eta:=1+\frac{1}{C_{0}}$. Then Corollary 2.6 yields

$$
\begin{aligned}
& B:=B\left(x_{0}, C_{0}\right) \Longrightarrow \operatorname{DS}(\eta B, 2, \alpha) \leq C, \\
& A:=A\left(x_{0}, r, \eta r\right), r \geq C_{0} \Longrightarrow \operatorname{DS}(\eta A, 2, \alpha) \leq C r^{1+\frac{\beta}{2}\left(\frac{1}{\alpha}-1\right)},
\end{aligned}
$$

where we recall that $\mu A\left(x_{0}, r, s\right):=A\left(x_{0}, \mu^{-1} r, \mu s\right)$ if $\mu>1, r<s$. The remainder of the proof is in three steps. In Step 0, we use Lemmas 2.9, 2.10 to establish weak Neumann-Poincaré inequalities, (2.11), (2.12), on slightly larger domains $\eta B_{\kappa}, \eta A_{\kappa}$, which together with (2.9), (2.10) imply weak Neumann-Sobolev inequalities (2.15), (2.16) for $\eta B, \eta A$. In Steps 1 and 2, we apply these four Neumann-type inequalities from Step 0, and Lemma 2.10 again, to prove (1.1) and (1.2), respectively. 
Step 0: Weak Neumann-type Poincaré and Sobolev on certain balls and annuli. For $\kappa \geq 1$ define $B_{\kappa}:=B\left(x_{0}, C_{0} \kappa\right)$ and $A_{\kappa}:=A\left(x_{0}, r, \eta \kappa r\right)$. We first show that

$$
\begin{aligned}
& \left\|u-u_{\eta B_{\kappa}}\right\|_{\eta B_{\kappa}, 2} \leq C(\kappa)\|\nabla u\|_{\eta^{2} B_{\kappa}, 2}, \\
& \left\|u-u_{\eta A_{\kappa}}\right\|_{\eta A_{\kappa}, 2} \leq C(\kappa) r\|\nabla u\|_{\eta^{2} A_{\kappa}, 2} .
\end{aligned}
$$

We write out a detailed argument for the annulus case (2.12) only. Pick a maximal $r / 2000 C_{0}$-separated set $x_{1}, \ldots, x_{m}$ in $\eta A_{\kappa}$, so that the $B_{i}:=B\left(x_{i}, r / 1000 C_{0}\right)$ cover $\eta A_{\kappa}$, but the $\frac{1}{2} B_{i}$ are disjoint. Notice that $\left|B_{i}\right| \sim r^{\beta}$ from $\operatorname{SOB}(\beta)$, so $m \leq C(\kappa)$. Then, from $\operatorname{SOB}(\beta)$ and the segment inequality (2.7), for all $i$ and $1 \leq \lambda \leq 10$,

$$
\int_{\lambda B_{i}}\left|u-u_{\lambda B_{i}}\right|^{2} \leq C r^{2} \int_{2 \lambda B_{i}}|\nabla u|^{2} .
$$

Then for any $\mu \in \mathbb{R}$,

$$
\int_{\eta A_{\kappa}}\left|u-u_{\eta A_{\kappa}}\right|^{2} \leq \int_{\eta A_{\kappa}}|u-\mu|^{2} \leq 2 \sum \int_{B_{i}}\left|u-u_{B_{i}}\right|^{2}+2 \sum\left|B_{i}\right|\left|u_{B_{i}}-\mu\right|^{2} .
$$

The first sum can be bounded by using (2.13) with $\lambda=1$. For the second, we apply Lemma 2.10, as follows. Construct a graph $G=(V, E)$ by setting $V:=\{1, \ldots, m\}$, and for $i \neq j,\{i, j\} \in E$ iff $B_{i} \cap B_{j} \neq \emptyset$. Then $G$ is connected because $\eta A_{\kappa}$ is. Let $w \equiv \frac{1}{m}$, and for $i \neq j$, let $\gamma^{\{i, j\}}$ be any path without loops joining $i$ and $j$. Thus, by Lemma 2.10, if $\mu:=\frac{1}{m} \sum u_{B_{i}}$, then the second sum in (2.14) is bounded by

$$
\sum\left|B_{i}\right|\left|u_{B_{i}}-\mu\right|^{2} \leq C r^{\beta} \sum_{i} \sum_{j: B_{i} \cap B_{j} \neq \emptyset}\left|u_{B_{i}}-u_{B_{j}}\right|^{2} .
$$

Next, for any constant $\nu \in \mathbb{R}$, by Cauchy-Schwarz,

$$
\begin{aligned}
\left|u_{B_{i}}-u_{B_{j}}\right|^{2} & \leq \frac{1}{\left|B_{i}\right|\left|B_{j}\right|} \int_{B_{i} \times B_{j}}|u(x)-u(y)|^{2} d x d y \\
& \leq 4 \frac{\left|B_{i} \cup B_{j}\right|}{\left|B_{i}\right|\left|B_{j}\right|} \int_{B_{i} \cup B_{j}}|u(x)-\nu|^{2} d x .
\end{aligned}
$$

Now $B_{i} \cup B_{j} \subset 3 B_{i}$ since $B_{i} \cap B_{j} \neq \emptyset$, so put $\nu=u_{3 B_{i}}$ and apply (2.13), $\lambda=3$ :

$$
\left|u_{B_{i}}-u_{B_{j}}\right|^{2} \leq C r^{2-\beta} \int_{6 B_{i}}|\nabla u|^{2} .
$$

This bounds the second sum in (2.14), concluding the proof of (2.12). The proof of (2.11) is entirely similar, dropping the $r$-dependence everywhere. Observe that we could apply Lemma 2.9 to $\eta B_{\kappa}$ directly in that case, but it will be convenient later on to be integrating over $\eta^{2} B_{\kappa}$ rather than $2 B_{\kappa}$ on the right-hand side of (2.11).

To conclude, we deduce weak Neumann-type Sobolev inequalities for $\eta B$ and $\eta A$. Construct cutoff functions $\chi_{B} \in C_{0}^{\infty}(\eta B), 0 \leq \chi_{B} \leq 1, \chi_{B} \equiv 1$ on $B,\left|\nabla \chi_{B}\right| \leq C$, and $\chi_{A} \in C_{0}^{\infty}(\eta A), 0 \leq \chi_{A} \leq 1, \chi_{A} \equiv 1$ on $A,\left|\nabla \chi_{A}\right| \leq C r^{-1}$. Then, setting $\kappa=1$, (2.9), (2.11) and (2.10), (2.12) easily imply

$$
\begin{aligned}
& \left\|u-u_{\eta B}\right\|_{B, 2 \alpha} \leq\left\|\chi_{B}\left(u-u_{\eta B}\right)\right\|_{\eta B, 2 \alpha} \leq C\|\nabla u\|_{\eta^{2} B, 2}, \\
& \left\|u-u_{\eta A}\right\|_{A, 2 \alpha} \leq\left\|\chi_{A}\left(u-u_{\eta A}\right)\right\|_{\eta A, 2 \alpha} \leq C r^{1+\frac{\beta}{2}\left(\frac{1}{\alpha}-1\right)}\|\nabla u\|_{\eta^{2} A, 2} .
\end{aligned}
$$

Together with (2.11), (2.12), these are what we need for Steps 1 and 2 below.

Put $r_{i}:=\eta^{i} C_{0}\left(i \in \mathbb{N}_{0}\right), A_{0}:=B=B\left(x_{0}, r_{0}\right), A_{i}:=A\left(x_{0}, r_{i-1}, r_{i}\right)(i \in \mathbb{N})$. Fix $\varphi \in C^{\infty}(M), \varphi>0$, to be determined, and use $\|. .\|_{X, \varphi, p}$ to denote the $L^{p}$ norm on $X$ with respect to $\varphi d$ vol. Let $u_{i}:=u_{\eta A_{i}}$ and $\varphi_{i}:=\sup _{A_{i}} \varphi\left(i \in \mathbb{N}_{0}\right)$. 
Step 1: Proof of (1.1). For $\mu \in \mathbb{R}$ to be determined, consider (all sums over $\mathbb{N}_{0}$ )

$$
\|u-\mu\|_{M, \varphi, 2 \alpha}^{2} \leq 2 \sum\left\|u-u_{i}\right\|_{A_{i}, \varphi, 2 \alpha}^{2}+2 \sum\left(\varphi_{i}\left|A_{i}\right|\right)^{\frac{1}{\alpha}}\left|u_{i}-\mu\right|^{2} .
$$

The first sum can be bounded by (2.15) for $i=0$ and (2.16), $A=A_{i}$, for $i \geq 1$ :

$$
\sum\left\|u-u_{i}\right\|_{A_{i}, \varphi, 2 \alpha}^{2} \leq C \sum \varphi_{i}^{\frac{1}{\alpha}} r_{i}^{2+\beta\left(\frac{1}{\alpha}-1\right)}\|\nabla u\|_{\eta^{2} A_{i}, 2}^{2} .
$$

For the second sum in (2.17), we apply Lemma 2.10 to the graph $A_{0}-A_{1}-A_{2}-\cdots$ with weights $w_{i}:=\tilde{w}_{i} / \tilde{w}$, where $\tilde{w}_{i}:=\left(\varphi_{i}\left|A_{i}\right|\right)^{1 / \alpha}$ and $\tilde{w}:=\sum \tilde{w}_{i}$, assuming that this series converges. Thus, if we choose

$$
\mu:=\sum w_{i} u_{i}=\int_{M} u \psi d \mathrm{vol}, \psi:=\sum \frac{w_{i}}{\left|\eta A_{i}\right|} \chi_{\eta A_{i}}, \int_{M} \psi d \mathrm{vol}=1,
$$

then we can continue to estimate the second sum in (2.17) as follows:

$$
\sum\left(\varphi_{i}\left|A_{i}\right|\right)^{\frac{1}{\alpha}}\left|u_{i}-\mu\right|^{2} \leq \frac{C}{\tilde{w}} \sum\left(\bar{w}_{k}+\bar{w}_{k+1}\right)\left|u_{k}-u_{k+1}\right|^{2}, \bar{w}_{k}:=\sum_{i \leq k \leq j}(j-i) \tilde{w}_{i} \tilde{w}_{j} .
$$

As before, for all $k \geq 0$ and any $\nu \in \mathbb{R}$, by Cauchy-Schwarz,

$$
\begin{aligned}
\left|u_{k}-u_{k+1}\right|^{2} & \leq \frac{1}{\left|\eta A_{k}\right|\left|\eta A_{k+1}\right|} \int_{\eta A_{k} \times \eta A_{k+1}}|u(x)-u(y)|^{2} d x d y \\
& \leq 4 \frac{\left|\eta A_{k} \cup \eta A_{k+1}\right|}{\left|\eta A_{k}\right|\left|\eta A_{k+1}\right|} \int_{\eta A_{k} \cup \eta A_{k+1}}|u(x)-\nu|^{2} d x .
\end{aligned}
$$

For $k=0$, apply (2.11), with $\kappa=\eta$, and for $k \geq 1$, (2.12), with $r=r_{k-1}, \kappa=\eta^{2}$, choosing $\nu$ to be the average of $u$ over the appropriate domain in each case. Thus, $\|u-\mu\|_{M, \varphi, 2 \alpha} \leq C\|\nabla u\|_{2}$ with $\mu$ as in (2.18), provided $\varphi: M \rightarrow \mathbb{R}^{+}$satisfies

$$
\sup _{i \in \mathbb{N}_{0}} \varphi_{i}^{\frac{1}{\alpha}} r_{i}^{2+\beta\left(\frac{1}{\alpha}-1\right)} \leq C, \frac{1}{C} \leq \tilde{w} \leq C, \sup _{k \in \mathbb{N}_{0}}\left(\bar{w}_{k}+\bar{w}_{k+1}\right) r_{k}^{2-\beta} \leq C .
$$

The first condition checks if $\varphi \leq C(1+r)^{\alpha(\beta-2)-\beta}$, the second if $\left.\varphi\right|_{A_{0}} \geq C^{-1}$ and $\varphi \leq C(1+r)^{-\beta-\varepsilon}(\varepsilon>0)$, and the third if $\varphi \leq C(1+r)^{\alpha(\beta-2)-\beta-\varepsilon}(\varepsilon>0)$.

Step 2: Proof of (1.2). We begin as in Step 1, splitting (and summing over $\mathbb{N}_{0}$ )

$$
\|u\|_{M, \varphi, 2 \alpha}^{2} \leq 2 \sum\left\|u-u_{i}\right\|_{A_{i}, \varphi, 2 \alpha}^{2}+2 \sum\left(\varphi_{i}\left|A_{i}\right|\right)^{\frac{1}{\alpha}}\left|u_{i}\right|^{2} .
$$

The first sum can be bounded by (2.15) for $i=0$ and (2.16), $A=A_{i}$, for $i \geq 1$ :

$$
\sum\left\|u-u_{i}\right\|_{A_{i}, \varphi, 2 \alpha}^{2} \leq C \sum \varphi_{i}^{\frac{1}{\alpha}} r_{i}^{2+\beta\left(\frac{1}{\alpha}-1\right)}\|\nabla u\|_{\eta^{2} A_{i}, 2}^{2} .
$$

To estimate the second sum, fix $K \in \mathbb{N}$ and consider

$$
\sum\left(\varphi_{i}\left|A_{i}\right|\right)^{\frac{1}{\alpha}}\left|u_{i}\right|^{2} \leq 2 \sum\left(\varphi_{i}\left|A_{i}\right|\right)^{\frac{1}{\alpha}}\left|u_{i}-u_{i+K}\right|^{2}+2 \sum\left(\varphi_{i}\left|A_{i}\right|\right)^{\frac{1}{\alpha}}\left|u_{i+K}\right|^{2} .
$$

The first term here can again be bounded in terms of the Dirichlet energy:

$$
\sum\left(\varphi_{i}\left|A_{i}\right|\right)^{\frac{1}{\alpha}}\left|u_{i}-u_{i+K}\right|^{2} \leq C K \sum\left(\varphi_{i}\left|A_{i}\right|\right)^{\frac{1}{\alpha}} r_{i}^{2-\beta} \int_{\eta^{2} A_{i} \cup \ldots \cup \eta^{2} A_{i+K}}|\nabla u|^{2} .
$$

To see this, write $u_{i}-u_{i+K}$ as a telescoping sum, use Cauchy-Schwarz, and estimate each term as in (2.19). As a result, if $\varphi \sim(1+r)^{\alpha(\beta-2)-\beta}$, then both (2.20), (2.22) 
are bounded by $C K^{2} \int|\nabla u|^{2}$. The remaining term in (2.21) can be absorbed:

$$
\begin{aligned}
\sum\left(\varphi_{i}\left|A_{i}\right|\right)^{\frac{1}{\alpha}}\left|u_{i+K}\right|^{2} & =\sum \frac{\left(\varphi_{i}\left|A_{i}\right|\right)^{\frac{1}{\alpha}}}{\left(\varphi_{i+K}\left|A_{i+K}\right|\right)^{\frac{1}{\alpha}}}\left(\varphi_{i+K}\left|A_{i+K}\right|\right)^{\frac{1}{\alpha}}\left|u_{i+K}\right|^{2} \\
& \leq C \eta^{(2-\beta) K} \sum\left(\varphi_{i}\left|A_{i}\right|\right)^{\frac{1}{\alpha}}\left|u_{i}\right|^{2},
\end{aligned}
$$

so it suffices to make $K$ sufficiently large, depending only on $\eta, \beta$, and $C$.

\section{Applichtions to the Poisson equation}

3.1. Existence. The basic idea in proving Theorem 1.5 is to solve boundary value problems on larger and larger domains. Then, by local elliptic theory, we only need a uniform $L^{\infty}$ bound to pass to the limit. To obtain such an estimate, we will apply Moser iteration based on Theorem 1.2. If $\beta>2$, the estimate becomes fairly simple thanks to (1.2). If $\beta \leq 2$, only (1.1) is available, and we need to assume $\int f=0$ in order to balance the subtraction on the left-hand side in (1.1).

This overall approach would not work for the complex Monge-Ampère equation because then we may not be able to solve any boundary value problems at all. The main idea in Tian-Yau [19] is to first solve $\left(\omega+i \partial \bar{\partial} u_{\varepsilon}\right)^{m}=e^{f+\varepsilon u_{\varepsilon}} \omega^{m}$ for all $\varepsilon>0$, which is substantially easier, and then exploit the exponential decay of $u_{\varepsilon}$.

- If $\beta>2$, suppose that $\Delta u=\left.f\right|_{\Omega}$ over a smooth and bounded domain $\Omega \subset M$, with $u=0$ on $\partial \Omega$, where $f \in C^{0, \alpha}(M)$ is as in the statement of Theorem 1.5. For $p>1$, multiply $\Delta u=f$ by $u|u|^{p-2}$ and integrate by parts to obtain

$$
\left.\left.\int_{\Omega}|\nabla| u\right|^{\frac{p}{2}}\right|^{2}=-\frac{p^{2}}{4(p-1)} \int_{\Omega} u|u|^{p-2} f .
$$

Define $\rho:=1+r, C_{0}:=\sup \rho^{\mu}|f|<\infty$, and $\|u\|_{p, \alpha}:=\left(\int_{M}|u|^{p} \rho^{\alpha(\beta-2)-\beta}\right)^{1 / p}$. By (3.1), (1.2), Hölder, and $\operatorname{SOB}(\beta)$, we then have the following two facts. First of all, for every $\alpha \in\left[1, \alpha_{n 2}\right]$ and $0<\varepsilon \leq \frac{1}{C}$ such that $\mu-2>\varepsilon(\alpha-1)$,

$$
p>1, p \geq \frac{\beta-2+\varepsilon}{\mu-2-\varepsilon(\alpha-1)} \Longrightarrow\|u\|_{\alpha p, \alpha} \leq \frac{C_{0} C p^{2}}{\varepsilon \alpha(p-1)},
$$

so this weighted $L^{\alpha p}$ norm is bounded independent of $\Omega$ and we can start to iterate at $\alpha p$. Second, for every $\alpha \in\left[1, \alpha_{n 2}\right]$ with $\alpha(\beta-2)>\beta-\mu$, and $0<\varepsilon \leq \frac{1}{C}$,

$$
p>1, p \geq \frac{\alpha(\beta-2+\varepsilon)}{\mu+\alpha(\beta-2)-\beta} \Longrightarrow\|u\|_{\alpha p, \alpha} \leq\left(\frac{C_{0} C p^{2}}{\varepsilon \alpha(p-1)}\right)^{\frac{1}{p}}\|u\|_{p, \alpha}^{1-\frac{1}{p}},
$$

so we can keep iterating from any such $p$. This proves Theorem 1.5 if $\beta>2$.

- If $\beta \leq 2$, suppose instead that $\Delta u=\left.f\right|_{\Omega}-\left(\left.f\right|_{\Omega}\right)_{\Omega}$ on $\Omega$ with $u=0$ on $\partial \Omega$. For any $\lambda \in \mathbb{R}$, the function $u+\lambda$ has the same Laplacian as $u$ and is equal to $\lambda$ on the boundary, which implies that (3.1) holds for $u+\lambda$ as well since $\int_{\Omega} \Delta u=0$. Now define $\rho$ and $C_{0}$ as before. For any $\delta>0$, put

$$
\|u\|_{p, \alpha, \delta}:=\left(\int_{M}|u|^{p} \rho^{\alpha(\beta-2-\delta)-\beta}\right)^{\frac{1}{p}},
$$

and recall the weight $\psi_{\delta} \sim \rho^{-2-\delta}$ needed for Theorem 1.2(i). Also, before iterating, observe that $f_{\Omega}$ can be controlled: If $B\left(x_{0}, r\right) \subset \Omega \subset B\left(x_{0}, 2 r\right)$ with $r \geq C$, then

$$
\left|f_{\Omega}\right| \leq \frac{1}{|\Omega|} \int_{M \backslash \Omega}|f| \leq C_{0} C r^{-\mu} .
$$


Then, by (3.1) with $p=2$ applied to $u-u_{\psi}$ as explained above, (3.3), and (1.1), if $\alpha \in\left[1, \alpha_{n 2}\right]$ and $0<\varepsilon \leq \frac{1}{C}$ satisfy $\mu-2>\varepsilon(\alpha-1)+\delta$ and $\varepsilon \leq \delta$, then

$$
\left\|u-u_{\psi_{\delta}}\right\|_{2 \alpha, \alpha, \delta} \leq \frac{C_{0} C_{\delta}}{\varepsilon \alpha} .
$$

Next, by (3.1) applied to $u-u_{\psi}$, but for $p \geq 2$, and by (1.1) again,

$$
\left\|\left|u-u_{\psi_{\delta}}\right|^{\frac{p}{2}}-\left(\left|u-u_{\psi_{\delta}}\right|^{\frac{p}{2}}\right)_{\psi_{\delta}}\right\|_{2 \alpha, \alpha, \delta}^{2} \leq \frac{C_{\delta} p^{2}}{p-1} \int_{\Omega}\left|u-u_{\psi_{\delta}}\right|^{p-1}\left|f-f_{\Omega}\right| .
$$

To proceed, we note the following simple, general inequality:

$$
\left\|\left(|v|^{\frac{p}{2}}\right)_{\psi_{\delta}}\right\|_{2 \alpha, \alpha, \delta} \leq\left(\int_{M} \psi_{\delta}\right)^{-1}\left(\int_{M} \frac{\psi_{\delta}^{2}}{\varphi_{\alpha, \delta}}\right)^{\frac{1}{2}}\left(\int_{M} \varphi_{\alpha, \delta}\right)^{\frac{1}{2 \alpha}}\|v\|_{p, \alpha, \delta}^{\frac{p}{2}},
$$

where $\varphi_{\alpha, \delta}:=\rho^{\alpha(\beta-2-\delta)-\beta}$ and the product of the three coefficients on the right is bounded by $C /(\varepsilon \alpha(2-\beta+\delta))$ if $1 \leq \alpha \leq 2-\varepsilon /(2-\beta+\delta)$ and $0<\varepsilon \leq \frac{1}{C}$. Then, combining (3.5) and (3.6), and assuming that $\alpha(2-\beta+\delta) \leq \mu-\beta$ and $\varepsilon \leq \delta$,

$$
\left\|u-u_{\psi_{\delta}}\right\|_{\alpha p, \alpha, \delta} \leq\left(\frac{C_{0} C_{\alpha, \delta, \varepsilon} p^{2}}{p-1}\right)^{\frac{1}{p}}\left\|u-u_{\psi_{\delta}}\right\|_{p, \alpha, \delta}^{1-\frac{1}{p}}+C_{\alpha, \delta, \varepsilon}^{\frac{1}{p}}\left\|u-u_{\psi_{\delta}}\right\|_{p, \alpha, \delta} .
$$

This concludes the proof as before. Observe that we were forced to start with $p=2$, but then on the plus side we obtain $\int|\nabla u|^{2}<\infty$ from the proof of (3.4).

3.2. Decay. We now prove Theorem 1.6 Assume $\Delta u=\left.f\right|_{\Omega}$ on a smooth bounded domain $\Omega$ with $u=0$ on $\partial \Omega$. Multiply the equation by $\zeta u|\zeta u|^{p-2} \zeta$, where $\zeta:=\rho^{l}$ for some fixed $l \in \mathbb{R}$, and $p>1$. Commute the spare factor of $\zeta$ past the Laplacian and integrate by parts. Using $|\nabla \rho|+\rho|\Delta \rho| \leq C$ and (1.2), for any $\alpha \in\left[1, \alpha_{n 2}\right]$,

$$
\left(\int \rho^{\alpha(\beta-2)-\beta}|\zeta u|^{\alpha p}\right)^{\frac{1}{\alpha}} \leq \frac{C p^{2}}{p-1}\left(\int|\zeta u|^{p-1} \zeta|f|+\frac{|l|(|l|+1) p}{p-1} \int \rho^{-2}|\zeta u|^{p}\right) .
$$

Let $C_{0}:=\sup \rho^{\mu}|f|<\infty$. Fix $p_{0}>1$ and let $p_{k}:=\alpha^{k} p_{0}$ and $\zeta_{k}:=\rho^{\alpha^{k}(\beta-2)-\beta}$ for $k \in \mathbb{N}$. Then, if $p_{0}(\mu-2) \geq \beta-2+\delta$ for some $0<\delta \leq \frac{1}{C}$, then, for all $k \in \mathbb{N}$,

$$
\left(\int \zeta_{k+1}|u|^{p_{k+1}}\right)^{\frac{1}{p_{k+1}}} \leq\left(\frac{C_{0} C_{\delta} p_{k}^{3}}{\left(p_{k}-1\right)^{2}}\right)^{\frac{1}{p_{k}}} \max \left\{1,\left(\int \zeta_{k}|u|^{p_{k}}\right)^{\frac{1}{p_{k}}}\right\} .
$$

The necessary bound for $\int \zeta_{1}|u|^{p_{1}}$ follows from (3.2) if $\alpha$ is close enough to 1 .

\section{ACKNOWLEDGMENTS}

I am grateful to my advisor, Gang Tian, for his constant support, and to Richard Bamler, Ronan Conlon, and Aaron Naber for helpful discussions. I would like to thank the anonymous referee for a careful reading of the paper and for pointing out a shortcut which made Section 2.1 much more readable.

\section{REFERENCES}

1. M. Anderson, The $L^{2}$ structure of moduli spaces of Einstein metrics on 4-manifolds, Geom. Funct. Anal. 2 (1992), 29-89. MR1143663 (92m:58017)

2. P. Buser, A note on the isoperimetric constant, Ann. Sci. École Norm. Sup. (4) 15 (1982), 213-230. MR683635 (84e:58076)

3. G. Carron, Inégalités de Hardy sur les variétés riemanniennes non-compactes, J. Math. Pures Appl. (9) 76 (1997), 883-891. MR1489943 (99c:53026) 
4. J. Cheeger, T. Colding, Lower bounds on Ricci curvature and the almost rigidity of warped products, Ann. of Math. (2) 144 (1996), 189-237. MR.1405949 (97h:53038)

5. C. Croke, Some isoperimetric inequalities and eigenvalue estimates, Ann. Sci. École Norm. Sup. (4) 13 (1980), 419-435. MR608287 (83d:58068)

6. S. Gallot, Inégalités isopérimétriques et analytiques sur les variétés riemanniennes, On the geometry of differentiable manifolds (Rome, 1986), 31-91, Astérisque 163-164 (1988). MR999971 (90f:58173)

7. A. Grigor'yan, L. Saloff-Coste, Stability results for Harnack inequalities, Ann. Inst. Fourier (Grenoble) 55 (2005), 825-890. MR2149405 (2006b:58026)

8. M. Gromov, Metric structures for Riemannian and non-Riemannian spaces, Progress in Mathematics, 152, Birkhäuser, Boston, MA, 1999. MR1699320(2000d:53065)

9. K. Hattori, The volume growth of hyper-Käehler manifolds of type $A_{\infty}$, J. Geom. Anal., to appear (Online First, 5 August 2010).

10. E. Hebey, Sobolev spaces on Riemannian manifolds, Lecture Notes in Mathematics, 1635, Springer-Verlag, Berlin, 1996. MR1481970 (98k:46049)

11. H.-J. Hein, On gravitational instantons, Ph.D. thesis, Princeton University, September 2010.

12. P. Li, Lecture notes on geometric analysis, available from http://math.uci.edu/ pli/.

13. P. Li, L.-F. Tam, Green's functions, harmonic functions, and volume comparison. J. Differential Geom. 41 (1995), 277-318. MR1331970 (96f:53054)

14. P. Maheux, L. Saloff-Coste, Analyse sur les boules d'un opérateur sous-elliptique, Math. Ann. 303 (1995), 713-740. MR1359957 (96m:35049)

15. J. Michael, L. Simon, Sobolev and mean-value inequalities on generalized submanifolds of $R^{n}$, Comm. Pure Appl. Math. 26 (1973), 361-379. MR0344978 (49:9717)

16. V. Minerbe, Weighted Sobolev inequalities and Ricci flat manifolds, Geom. Funct. Anal. 18 (2009), 1696-1749. MR2481740 (2010g:53064)

17. P. Petersen, Convergence theorems in Riemannian geometry, Comparison geometry (Berkeley, 1993-94), 167-202, MSRI Publ., 30, Cambridge University Press, Cambridge, 1997. MR.1452874 (98k:53049)

18. L. Saloff-Coste, Aspects of Sobolev-type inequalities, London Mathematical Society Lecture Note Series, 289, Cambridge University Press, Cambridge, 2002. MR1872526 (2003c:46048)

19. G. Tian, S.-T. Yau, Complete Kähler manifolds with zero Ricci curvature, I, J. Amer. Math. Soc. 3 (1990), 579-609. MR.1040196 (91a:53096)

20. G. Tian, S.-T. Yau, Complete Kähler manifolds with zero Ricci curvature, II, Invent. Math. 106 (1991), 27-60. MR.1123371 (92j:32028)

21. C. van Coevering, Regularity of asymptotically conical Ricci-flat Kähler metrics, arXiv: 0912.3946 .

Department of Mathematics, Imperial College London, London SW7 2AZ, United KINGDOM

E-mail address: h.hein@imperial.ac.uk 